\title{
The effect of phytohormones on the growth, cellulose production and pellicle properties of Gluconacetobacter xylinus ATCC 53582
}

\author{
Osama Qureshi, ${ }^{1}$ Hira Sohail, ${ }^{1}$ Andrew Latos,, ${ }^{1,2}$ Janice L. Strap ${ }^{1}$ \\ ${ }^{1}$ Faculty of Science, University of Ontario Institute of Technology, Oshawa; ${ }^{2}$ Faculty of Dentistry, \\ University of Toronto, Ontario, Canada
}

\begin{abstract}
Gluconacetobacter xylinus is a plant-associated bacterium best studied for its cellulose production. Bacterial cellulose is important in facilitating plant-microbe interactions but little is known about the effect that exogenous phytohormones have on bacterial cellulose synthesis or the growth of $G$. xylinus. We characterized the growth, development and effect on pellicle characteristics caused by exogenous indole-3acetic acid (IAA), gibberellic acid (GA), abscisic acid (ABA) and zeatin (Z) over a range of concentrations $(1 \mathrm{nM}$ to $100 \mu \mathrm{M})$. These phytohormones are plant growth regulators known to be involved plant development including fruit ripening and stress tolerance. Each of these hormones stimulated $G$. xylinus growth and influenced its pellicle charac-
\end{abstract}

Correspondence: Janice L. Strap, Faculty of Science, UA 4055, 2000 Simcoe Street North, University of Ontario Institute of Technology, Oshawa, Ontario, Canada L1H 7K4.

Tel. +1.905.721.8668 ext. 2935 - Fax: +1.905.721.3304.

E-mail: janice.strap@uoit.ca

Key words: cellulose, phytohormones, abscisic acid, gibberellic acid, indole3-acetic acid, zeatin, crystallinity, Gluconacetobacter xylinus.

Acknowledgements: the authors wish to thank F. Gaspari for assistance with FTIR.

Conference presentation: part of this paper was presented at the $3^{\text {rd }}$ International Conference on Acetic Acid Bacteria. Vinegar and Other products, 2012 Apr 17-20, Cordoba, Spain (http://www.uco.es/aab2012/).

Funding: UOIT-Start-up funds and Natural Sciences and Engineering Research Tools and Instruments (NSERC-RTI) grant to J.L. Strap.

Contributions: JLS conceived and designed the experimental work, contributed to the experimental work and co-wrote the manuscript; OQ, HS, AL carried out experimental work and contributed to writing the manuscript.

Conflict of interests: the authors declare no potential conflict of interests.

Received for publication: 1 August 2012.

Revision received: 14 October 2012.

Accepted for publication: 18 October 2012.

This work is licensed under a Creative Commons Attribution 3.0 License (bync 3.0).

(C) Copyright O. Qureshi et al., 2013

Licensee PAGEPress, Italy

Acetic Acid Bacteria 2013; 2(s1):e7

doi:10.4081/aab.2013.s1.e7 teristics. Exogenous IAA had the greatest effect on $G$. xylinus pellicles. Growth in IAA produced thin pellicles with very little cellulose. In general, pellicle wet weight was inversely proportional to the bacterial cellulose yield when cultures were grown in the presence of ABA, suggesting ABA influenced pellicle density and hydration. The crystallinity index, CI (IR) of cellulose produced in the presence of each phytohormone over a variety of concentrations was determined by Fourier transform infrared spectroscopy. The observed effect on cellulose crystallinity was concentration and hormone dependent. GA caused the greatest alterations in crystallinity with the highest $\mathrm{CI}(\mathrm{IR})=0.94$ at 1 $\mu \mathrm{M}$ and the lowest $\mathrm{CI}(\mathrm{IR})=0.47$ at $500 \mathrm{nM}$. Endogenous production of hormones by $G$. xylinus was investigated by high performance liquid chromatography of extracts prepared from both cell pellets and culture supernatants. We found $G$. xylinus synthesized GA, ABA and Z but did not produce IAA.

\section{Introduction}

Gluconacetobacter xylinus is a gram-negative bacterium that can be isolated from rotting fruits and vegetables ${ }^{1,2}$ however the mechanisms involved in this plant-microbe interaction are not known in detail. In vitro, G. xylinus produces a cellulose pellicle at the air/liquid interface of broth-grown cultures. The cellulose biofilm produced by this organism has been the subject of intense investigation due to its purity, mechanical strength, and high crystallinity. ${ }^{3-11}$ These properties make bacterial cellulose useful for a variety of industrial applications. . $^{3,12-15}$ Despite decades of study, the complete cellulose biosynthetic pathway has not yet been elucidated; there is still much to learn about its regulation, export mechanisms and assembly into the crystallized form.

We recently identified a small molecule, pellicin, capable of disrupting crystalline cellulose formation by $G$. xylinus. ${ }^{16}$ Given the importance of bacterial cellulose for plant-microbe interactions ${ }^{17-20}$ it was of interest to investigate whether phytohormones are also capable of influencing bacterial cellulose crystallinity as this will have implications for attachment, growth and survival of plant-associated microbes.

In this study, representative phytohormones known to play a key role in ripening and senescence of fruits and vegetables were chosen: gibberellic acid (GA), abscisic acid (ABA), the auxin indole-3-acetic acid (IAA), and the cytokinin zeatin. In plants, auxins stimulate cell wall synthesis, cell enlargement, cell division and importantly delay leaf senescence and fruit ripening. ${ }^{21}$ At high concentrations, IAA has been shown to reduce root growth. ${ }^{22}$ Gibberellins, a large class of phytohormone comprised of more than one hundred compounds, ${ }^{23}$ have been implicated in the stimulation of both cell division and cell elongation in plant stems ${ }^{22,24}$ and serves an important role in primary root growth by regulating cellulose deposition. ${ }^{25}$ In grapes, exogenous application of gibberellins induce fruit setting and fruit growth. ${ }^{24}$ Cytokinins are 
adenine derivatives that function in many aspects of plant growth and development including the induction of cell division in fruits and shoots as well as the delay of senescence. ${ }^{26,27} \mathrm{ABA}$ opposes the activity of the other classes of phytohormone exerting an inhibitory effect on plant growth. $\mathrm{ABA}$ is also involved in fruit ripening. ${ }^{28}$ Moreover, exogenous ABA treatment of grapevines has been shown to enhance the activity of cellulase promoting the decomposition of cellulose ${ }^{29}$ leading to fruit softening.

While there have been many reports on the effect of bacterially produced phytohormone-like compounds on plants, ${ }^{30-35}$ very few studies have focussed on the effect that plant hormones have on the growth and biofilm production of bacteria. Therefore, the purpose of the present study was to examine the effect that phytohormones have on the growth and development of Gluconacetobacter xylinus ATCC 53582 and in particular, to determine the effect that these hormones have on pellicle characteristics.

\section{Materials and Methods}

\section{Chemicals}

Zeatin (Z) and ABA were purchased from BioBasic (Markham, ON, Canada). GA and IAA were purchased from BioShop (Burlington, ON, Canada).

\section{Bacteria, growth medium and culture conditions}

Gluconacetobacter xylinus ATCC 53582 was grown in SchrammHestrin (SH) medium ${ }^{8}$ either on a rotary shaker at $30^{\circ} \mathrm{C}$ or under static conditions. To obtain a uniform suspension of $G$. xylinus cells lacking cellulose, 0.1\% (v/v) filter-sterilized cellulose (Sigma, St. Louis, MO, USA) was added to cultures. Growth curve data was obtained in 96well microtitre plates inoculated with a total volume of $150 \mu \mathrm{L} \mathrm{SH}$ medium containing $0.1 \%$ cellulose and either dimethylsulfoxide (DMSO) or $\mathrm{ABA}, \mathrm{GA}$, IAA or Z dissolved in DMSO and appropriately diluted in $\mathrm{SH}$ broth to give concentrations of $0-100 \mu \mathrm{M}$. The addition of DMSO does not alter the growth rate of $G$. xylinus under the growth conditions used in this study. ${ }^{16}$ The inoculum was prepared by harvesting 3 -day old, cellulase-digested $G$. xylinus cultures by centrifugation at $3000 \mathrm{x} g$ for 10 min at $4^{\circ} \mathrm{C}$, washing once with $0.1 \%$ saline and twice in room temperature $\mathrm{SH}$ broth. The starting inoculum was adjusted to an optical density at $600 \mathrm{~nm}$ of 0.05 in SH broth. Culture plates were incubated at $30^{\circ} \mathrm{C}$ with shaking at $150 \mathrm{rpm}$. Optical density was recorded at $600 \mathrm{~nm}$ using a Bio-Rad xMark ${ }^{\mathrm{TM}}$ Microplate Absorbance Spectrophotometer (Bio-Rad Laboratories Ltd., Mississauga, ON, USA). Growth was followed for 204 h. Data from three biological replicates each with six technical replicates were averaged. Growth rates were calculated for each treatment and normalized against $G$. xylinus grown in the absence of phytohormone to facilitate comparison.

\section{Pellicle characteristics}

To investigate the effect of phytohormones on the $G$. xylinus pellicle, the bacterium was cultivated under static conditions at $30^{\circ} \mathrm{C}$ in 24 -well plates containing $2 \mathrm{~mL} \mathrm{SH}$ medium supplemented with either ABA, GA, IAA or $\mathrm{Z}$ in concentrations ranging from $1 \mathrm{nM}$ to $100 \mu \mathrm{M}$. The inoculum was prepared and standardized as described above. All media had an initial $\mathrm{pH}$ of 6.3. Three biological replicates, each with six technical replicates were used for data collection. The $\mathrm{pH}$ of culture supernatants was recorded after pellicle removal at the end of seven days of static growth.

The thickness of $G$. xylinus pellicle was measured without the removal of water at the time of harvest for each treatment. Pellicles from each replicate were photographed from the side and the thickness measured using Macnification software (Orbicule, Inc, Belgium) at three different positions on each pellicle; the values were averaged.

Bacterial cellulose yield was determined by first recording the pellicle wet weights and then treating with $0.1 \mathrm{~N} \mathrm{NaOH}$ at $80^{\circ} \mathrm{C}$ for $20 \mathrm{~min} .{ }^{36}$ Treated pellicles were washed in water until neutral, then dried in an oven at $55^{\circ} \mathrm{C}$ to constant weight.

Crystallinity of pellicles formed by $G$. xylinus under each of the phytohormone treatments was assessed using Fourier transform infrared (FTIR) spectroscopy. Replicate pellicles from each treatment that had been $\mathrm{NaOH}$-treated, washed and dried were used for FTIR analysis on a Perkin Elmer Precisely Spectrum 100 FTIR spectrometer with an horizontal attenuated total reflectance sampling accessory in triplicate using 10 scans with a resolution of $4 \mathrm{~cm}^{-1}$ for each measurement in the range of 4000 to $650 \mathrm{~cm}^{-1}$. Background correction was performed prior to collecting sample data. The crystallinity index, CI (IR) was calculated as $\mathrm{A}_{1437} / 895$ as previously described. ${ }^{37}$

\section{Detection of endogenous $G$. xylinus hormones}

Gluconacetobacter xylinus was grown in $\mathrm{SH}$ medium with and without $0.05 \%(\mathrm{w} / \mathrm{v})$ L-tryptophan under agitated conditions. The cellulose pellicle was digested overnight by addition of $0.1 \%(\mathrm{v} / \mathrm{v})$ filter-sterilized cellulase. The cultures were then centrifuged at $3000 \mathrm{x} g$ for $15 \mathrm{~min}$ at room temperature. Extraction and detection of the free and the bound forms of abscisic acid, GA, IAA and Z from $G$. xylinus cell pellets and culture supernatants were performed as previously described ${ }^{31,38}$ with minor modifications. Briefly, the culture supernatant was transferred to a separate glass vial whereas the cell pellets were washed in $0.85 \%$ sterile saline solution and resuspended in $10 \mathrm{~mL}$ sterile water in a glass vial. The cell fraction was extracted with $20 \mathrm{~mL}$ of methanol:chloroform:ammonium hydroxide (12:5:3 v/v/v). The aqueous phase was centrifuged at $3000 \mathrm{x} \mathrm{g}$ for $15 \mathrm{~min}$ to remove cell debris. The clarified aqueous phase was transferred to a clean glass vial. The cell-derived aqueous phase and the culture supernatants were adjusted to $\mathrm{pH} 2.5$ and extracted three times with ethyl acetate to obtain a final volume of $25 \mathrm{~mL}$; the organic fraction contains the free form of IAA, GA and abscisic acid. The aqueous phase was then adjusted to $\mathrm{pH} 7$ and the samples were extracted three times with ethyl acetate. The organic fraction was expected to contain free $\mathrm{Z}$. The resultant aqueous phase was adjusted to $\mathrm{pH} 11$, hydrolyzed for $1 \mathrm{~h}$ at $70^{\circ} \mathrm{C}$, cooled to room temperature, then the $\mathrm{pH}$ was reduced to 7 and extracted three times with ethyl acetate. The organic fraction was expected to contain the bound form of $\mathrm{Z}$, if present. Next, the $\mathrm{pH}$ of the aqueous layer was reduced to $\mathrm{pH} 2.5$ to extract the bound forms of IAA, GA and ABA in the ethyl acetate fraction. Once extractions were complete, the samples were evaporated to dryness, solubilized in $2 \mathrm{~mL}$ of $30 \%$ acetonitrile, filtered through $0.2 \mathrm{~mm}$ filters and analyzed using a Shimadzu Prominence highperformance liquid chromatography (HPLC) system equipped with a photodiode array detector using a $5 \mathrm{~mm}$ apHera ${ }^{\mathrm{TM}} 150 \times 4.6 \mathrm{~mm} \mathrm{C18}$ column (Sepulco) at a flow rate of $0.8 \mathrm{~mL} / \mathrm{min}$ with isocratic elution in acetonitrile-water (26:74). Standard solutions of (+/-) ABA, GA, IAA and Z were used as reference. Absorbance was monitored at $265 \mathrm{~nm}$ for ABA and Z, $280 \mathrm{~nm}$ for IAA and $208 \mathrm{~nm}$ for GA as described previously. 31,39

Culture supernatants of $G$. xylinus grown in either SH or SH amended with $0.05 \%(w / v)$ L-tryptophan were assayed for the production of IAA using the method of Gordon and Weber ${ }^{40}$ as modified by Sarwar and Kremer ${ }^{41}$ in which culture filtrate was reacted with Salkowski reagent in a microplate. A pink-colored product after 30 min was taken to be a positive result. Quantitation of IAA equivalents was determined by reading the absorbance at $530 \mathrm{~nm}$.

\section{Microscopy}

Colonial morphology changes due to varying concentrations of phytohormones were assessed using an Amscope dissecting microscope equipped with a digital camera. 


\section{Statistical analysis}

All experiments were performed in triplicate. Each biological replicate was comprised of six technical replicates. The statistical analysis was performed using regular two-way ANOVA with Bonferroni posttests to compare the means. The analysis was performed using GraphPad Prism software, version 5.0 (GraphPad Software, La Jolla, CA, USA). The significance level reported is $\mathrm{P}<0.05$.

\section{Results}

\section{Growth of $G$. xylinus in broth cultures is affected by plant hormones}

Gluconacetobacter xylinus was grown in liquid culture in increasing concentrations of ABA, GA, IAA, and Z (Figure 1). G. xylinus grew to higher optical densities when grown in media containing phytohormone for all concentrations tested compared to untreated controls. The growth rates of $G$. xylinus grown in the presence of $100 \mathrm{nM}, 500 \mathrm{nM}$, $5 \mu \mathrm{M}$, and $50 \mu \mathrm{M}$ were significantly higher $(\mathrm{P}<0.05)$ than for cultures grown in the absence of ABA (Figure $1 \mathrm{~A}$ and $\mathrm{B}$ ). Cultures grown under these concentrations of ABA grew 1.45 times faster than untreated cultures. Very low concentrations ( $1 \mathrm{nM}-50 \mathrm{nM})$ of ABA had no significant effect on growth rate.

GA-grown cultures reached stationary phase more quickly than cultures grown in any of the other plant hormones tested, reaching the end of logarithmic phase at $110 \mathrm{~h}$ (Figure 1C). GA increased the growth rates of $G$. xylinus at all concentrations, but a significant increase was observed for $5 \mathrm{nM}$ to $50 \mathrm{nM}, 500 \mathrm{nM}, 5 \mu \mathrm{M}, 50 \mu \mathrm{M}$ and $100 \mu \mathrm{M}$. The greatest stimulation of growth rate was observed for $5 \mathrm{nM}$, which increased the growth rate 1.6 times that of control cultures, while for $50 \mathrm{nM}$ stimulated cultures to grow 1.5 times faster than control cultures (Figure 1D).

Notably, cultures grown in $100 \mu \mathrm{M}$ IAA started to lyse by $150 \mathrm{~h}$ (Figure 1E); such a precipitous decline in culture health was not observed for any other phytohormone treatment. The growth rates of cultures supplemented with IAA were significantly stimulated at concentrations of $5 \mathrm{nM}, 100 \mathrm{nM}, 5 \mu \mathrm{M}, 10 \mu \mathrm{M}$ and $100 \mu \mathrm{M}$ with the latter having the greatest effect by causing an increase in the growth rate to 1.5 times that of control cultures (Figure 1F).

$\mathrm{Z}$ significantly increased the growth of $G$. xylinus (Figure $1 \mathrm{G}$ ). Z stimulated the growth rate of cultures at concentrations of $1 \mathrm{nM}$ to 50 $\mathrm{nM}, 5 \mu \mathrm{M}$ and $50 \mu \mathrm{M}$ by $1.6,1.4,1.5$ and 1.4 times that of untreated control cultures, respectively (Figure $1 \mathrm{H}$ ).

\section{Effect of phytohormones on $G$. xylinus pellicle formation}

The effect that select phytohormones had on the bacterial cellulose production of $G$. xylinus was determined by examining pellicle thickness, wet weight and cellulose yield. To facilitate comparisons between treatments, measurements were normalized against pellicle properties derived from untreated cultures. The only concentration of ABA to significantly affect pellicle thickness was $50 \mathrm{nM}$ (Figure 2A). At this concentration, pellicles were 0.76 times thinner than pellicles produced by control cultures. The thinnest pellicles were observed at a GA concentration of $100 \mu \mathrm{M}$ while $5 \mathrm{nM}$ GA caused $G$. xylinus to produce pellicles 1.2 times thicker than pellicles produced by untreated cultures (Figure 2A). IAA had the most pronounced effect on pellicle thickness with most concentrations resulting in thinner pellicles compared to controls. IAA treatment resulted in thinner pellicles (Figure 2A), that were less hydrated (Figure 2B) and that contained the least cellulose (Figure 2C) of all the phytohormone treatments. In contrast, $\mathrm{Z}$ had no significant effect on pellicle thickness (Figure 2A) but did significantly affect the wet pellicle weight at low concentrations ranging from $10 \mathrm{nM}$ to $10 \mu \mathrm{M}$
(Figure 2B). ABA caused G. xylinus to produce pellicles of greater wet weight compared with the other three hormones (Figure 2B). Bacterial cellulose yield was only increased in ABA-grown cultures at concentrations of $1 \mathrm{nM}, 5 \mathrm{nM}$ and $500 \mathrm{nM}$ with the maximal yield being 1.12 times that of the cellulose yield obtained for control cultures. The cellulose yield for cultures grown in the presence of GA were only significantly affected for three concentrations. At $5 \mu \mathrm{M}$, the cellulose yield was 1.2 times that of controls, at $1 \mu \mathrm{M}$ the yield was 1.1 times that of control and at $100 \mu \mathrm{M}$, the yield dropped to 0.9 times that of the control cellulose yield (Figure 2C). Only a single concentration of Z, $10 \mathrm{nM}$, had a significant effect on bacterial cellulose yield (Figure 2C).

To examine whether extracellular $\mathrm{pH}$ changed in response to plant hormone treatment, the final $\mathrm{pH}$ of $G$. xylinus culture supernatants of cultures grown under static conditions in the presence and absence of plant hormones was measured. In general, the $\mathrm{pH}$ of culture supernatants after seven days of static growth was lower for higher concentrations of hormone, from $10 \mu \mathrm{M}$ to $100 \mu \mathrm{M}$ compared to untreated cultures. The converse was observed for low concentrations, between $1 \mathrm{nM}$ and $50 \mathrm{nM}$, which were observed to have higher $\mathrm{pH}$ compared to cultures grown in the absence of phytohormone (Figure 3).

\section{Structural characterization of pellicles produced in the presence of phytohormones}

To characterize the influence phytohormone treatment had on the structure of cellulose produced by $G$. xylinus, we calculated the crystallinity index, CI (IR) based on FTIR spectra. ${ }^{37}$ The CI (IR) of pellicles produced in the absence of phytohormone was 0.8 . ABA, GA, IAA and Z affected the crystallinity of pellicles formed in their presence in a concentration-dependent manner. There was an inverse relationship observed for the crystallinity of pellicles produced in the presence of $\mathrm{Z}$ and the crystallinity of pellicles produced in the presence of GA. At concentrations of $\mathrm{Z}$ that decrease the pellicle crystallinity, the crystallinity of GA-produced pellicles was greater. For example, at $500 \mathrm{nM}$, the CI (IR) of $\mathrm{Z}$ was 0.87 while for GA the CI (IR) was 0.47 , the lowest crystallinity observed for hormone-treated pellicles (Figure 4). ABA had the least effect on pellicle crystallinity except for intermediate concentrations (50 nM to $100 \mathrm{nM}$ ) which exhibited a decreased CI (IR) of 0.55 (Figure 4).

\section{Morphological changes caused by growth of $G$. xylinus on phytohormones}

The colonial morphology of $G$. xylinus was profoundly affected by growth on solid medium supplemented with phytohormones (Figure 5). Colonies of $G$. xylinus formed in the absence of exogenous phytohormones were $\sim 0.5 \mathrm{~cm}$ in diameter, irregular in form, off-white in color, convex in elevation and were filiform in margin. Overall, colonies were larger $(\geq 1 \mathrm{~cm}$ ) when grown in the presence of $>500 \mathrm{nM}$ phytohormone. Cellulose formation on solid medium was affected by the presence of phytohormone as well. For example, at $500 \mathrm{nM}, \mathrm{ABA}$ and IAA grown $G$. xylinus did not produce cellulose but rather formed smooth colonies. Cultures grown on GA barely produced cellulose at $500 \mathrm{nM}$ while Zgrown colonies exhibited more pronounced cellulose production (Figure 5). Colonies formed in the presence of $500 \mathrm{nM}$ ABA were pearlescent compared to colonies formed in the presence of the other phytohormones. The form of $G$. xylinus colonies at $500 \mathrm{nM}$ GA and IAA were round compared to the irregular form observed in the presence of the other phytohormones. The elevation of the colonies varied depending on exposure to phytohormone. For example, unexposed colonies were convex as were colonies formed in the presence of $\mathrm{Z}$, but were raised in the presence of $500 \mathrm{nM} \mathrm{GA}$, crateriform on $500 \mathrm{nM}$ IAA and flat on $500 \mathrm{nM}$ ABA. The margin of colonies in $500 \mathrm{nM}$ IAA were entire but were undulate for $\mathrm{ABA}$ and $\mathrm{Z}$ at the same concentration; this differed 

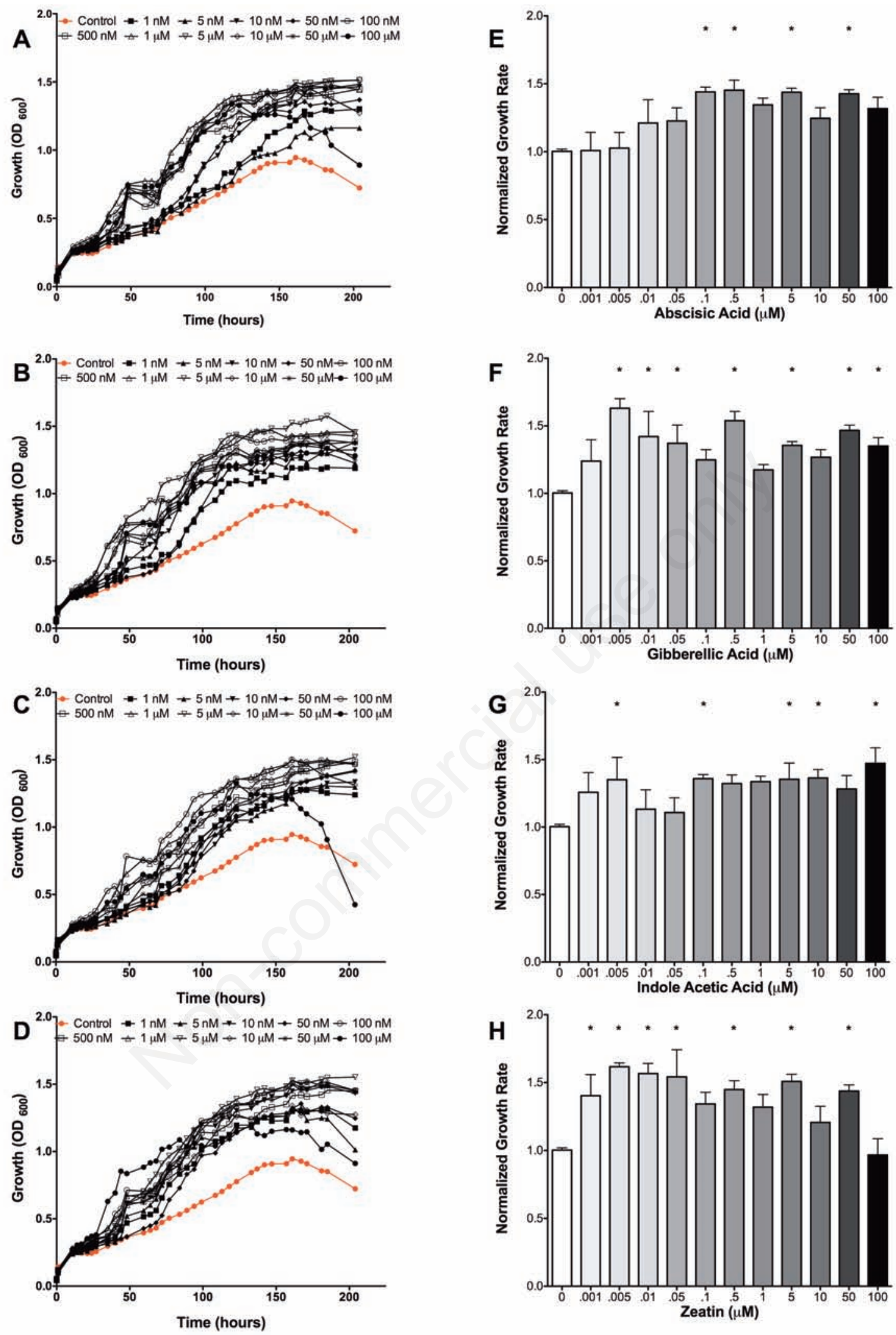

Figure 1. Phytohormones stimulate the growth of G. xylinus. Phytohormones did not affect the viability of G. xylinus when grown at $30^{\circ} \mathrm{C}$ in Schramm-Hestrin broth containing $0.1 \%$ cellulase in the presence and absence of phytohormones. Growth curves of $G . x y l i$ nus containing varying concentrations of $(A)$ abscisic acid, (B) gibberellic acid, (C) indole-3-acetic acid, and (D) zeatin. Growth rate of G. xylinus in the presence of varying concentrations of (E) abscisic acid, (F) gibberellic acid, (G) indole-3-acetic acid, and (H) zeatin normalized to the growth rate calculated for untreated cultures. Data show the mean for three biological replicates each with six technical replicates. Error bars shown are the standard error of the mean. Statistical analysis was performed using ANOVA with Bonferroni post-tests to compare the means. The significance level reported is $\mathbf{P}<0.05\left(^{*}\right)$. 
from the filiform margins observed for the untreated $G$. xylinus and for colonies formed in the presence of GA.

\section{Endogenous hormone production by G. xylinus}

In this study, exogenous phytohormones were found to affect the growth, colonial morphology, and pellicle characteristics of $G$. xylinus. Since this is a plant-associated bacterium, we sought to determine whether this bacterium endogenously produces any phytohormone-like compounds. Under the culture conditions used in this study, no IAA was detected in either cell pellets or culture supernatants of $G$. xylinus by colorimetric assays or after extraction and HPLC analysis. Free GA (Figure 6A) free $\mathrm{ABA}$ and free $\mathrm{Z}$ (Figure $6 \mathrm{~B}$ ) were detected. To our knowledge, this is the first report of endogenous $\mathrm{GA}, \mathrm{ABA}$ and $\mathrm{Z}$ production by $G$. xylinus.

\section{Discussion}

Bacteria involved in plant-microbe interactions have been reported to synthesize hormones such as ABA, GA, IAA and Z. ${ }^{27,33,42,43}$ The functional role of hormone synthesis by microorganisms is not fully understood. Even less is known about the influence exogenous phytohormones exert on bacterial growth and development or their impact on plant-microbe interactions. We report here that exogenous phytohormones influence the growth, development and pellicle characteristics of $G$. xylinus, including cellulose crystallinity. Furthermore, we found that under the culture conditions used in this study, G. xylinus ATCC 53582 produced endogenous GA, ABA and Z. Notably, IAA was not detected in either cells or culture supernatants.

Gluconacetobacter xylinus is known to be associated with rotting fruits. ${ }^{1,2}$ The phytohormones chosen in this study play a role in the ripening and senescence of fruits and vegetables. In plants, ABA serves to induce senescence while auxins, giberellins and cytokinins can delay senescence in a concentration-dependent manner. ${ }^{28,44-46}$ IAA is a plant growth promoting hormone synthesized by many plant growth promoting bacteria including Gluconacetobacter diazotrophicus. ${ }^{35,47}$ In contrast to G. diazotrophicus, G. xylinus does not synthesize IAA; however, exogenously supplied, this hormone greatly influenced growth and development. We observed that when $G$. xylinus was grown in the presence of IAA, pellicles were thin (Figure 2A) and of low cellulose content (Figure 2C). This result can be explained in context of a plantmicrobe interaction: elevated auxin levels in the plant delay ripening and senescence, ${ }^{28,48-50}$ as a result, the sugars available for bacterial cellulose synthesis will be lower compared to ripened fruit. Exogenous IAA impedes $G$. xylinus cellulose synthesis yet stimulates growth rate so that cellular density is at a peak when the fruit ripens and glucose becomes more readily available. During ripening, IAA production ceas$\mathrm{es},{ }^{51}$ alleviating the inhibition of bacterial cellulose synthesis. For example, immature strawberry seeds secrete auxins but when ripening starts IAA production stops ${ }^{51}$ We further speculate that $G$. xylinus may metabolize exogenous IAA reducing its effective concentration so that ripening can commence. There are several examples of plant-associated bacteria capable of degrading auxins. ${ }^{33,52,53}$ The capacity of $G$. xylinus to utilize auxins as carbon source will be explored in future investigations.

GA stimulated the growth of G. xylinus (Figure 1). Growth stimulation by this hormone has been observed for other bacteria including Azotobacter, Pseudomonas and Lactobacillus. ${ }^{23}$ At higher concentrations, exogenous GA increased the bacterial cellulose wet weight (Figure 2B) and yield (Figure 2C). Furthermore, GA had profound effect on the crystallinity of $G$. xylinus pellicles, depending on concentration. GA has been shown to stimulate endo- $\beta-1,4$-xylanase activity
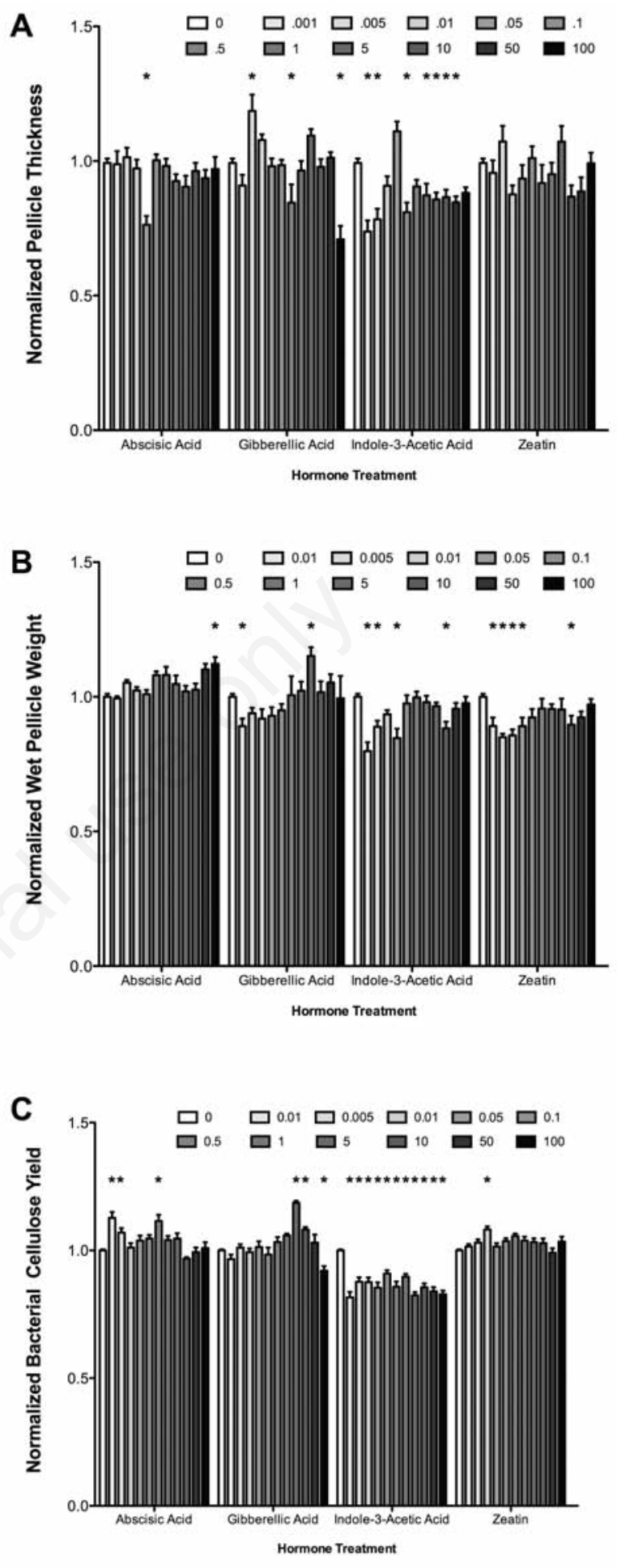

Figure 2. Phytohormones influence the physical pellicle characteristics and bacterial cellulose yield of G. xylinus. G. xylinus was grown under static conditions at $30^{\circ} \mathrm{C}$ in Schramm-Hestrin broth for 7 days in the presence and absence of the phytohoromones abscisic acid, gibberellic acid, indole-3-acetic acid, and zeatin. (A) Normalized pellicle thickness, (B) normalized pellicle wet weight, and $(C)$ normalized bacterial cellulose yield. The data is normalized to the data obtained for untreated cultures to facilitate comparisons. Data show the mean for three biological replicates each with six technical replicates. Error bars shown are the standard error of the mean. Statistical analysis was performed using ANOVA with Tukey post-tests to compare the means. The significance level reported is $\mathrm{P}<0.05\left(^{*}\right)$. 
in barley ${ }^{54}$ leading to the solubilisation of stored carbohydrate. We found that $G$. xylinus is capable of endogenous synthesis of GA (Figure 6). Notably, G. diazotrophicus, a nitrogen-fixing acetic acid bacterium synthesizes this hormone as well but synthesis is carbon sourcedependent. $^{34}$

$\mathrm{Z}$ stimulated the growth (Figure 1), pellicle thickness (Figure 2A), bacterial cellulose yield (Figure 2C) and crystallinity (Figure 4).
Interestingly, we observed an inverse relationship between the crystallinity of pellicles produced in the presence of $\mathrm{Z}$ and in the presence of GA. The physiological implications of this relationship for cellulose producing plant-associated bacteria require further investigation.

While there is a paucity of information in the literature on the effect that phytohormones have on bacterial growth, IAA and GA were both found to stimulate the growth of Azospirillum sp.; the extent of growth

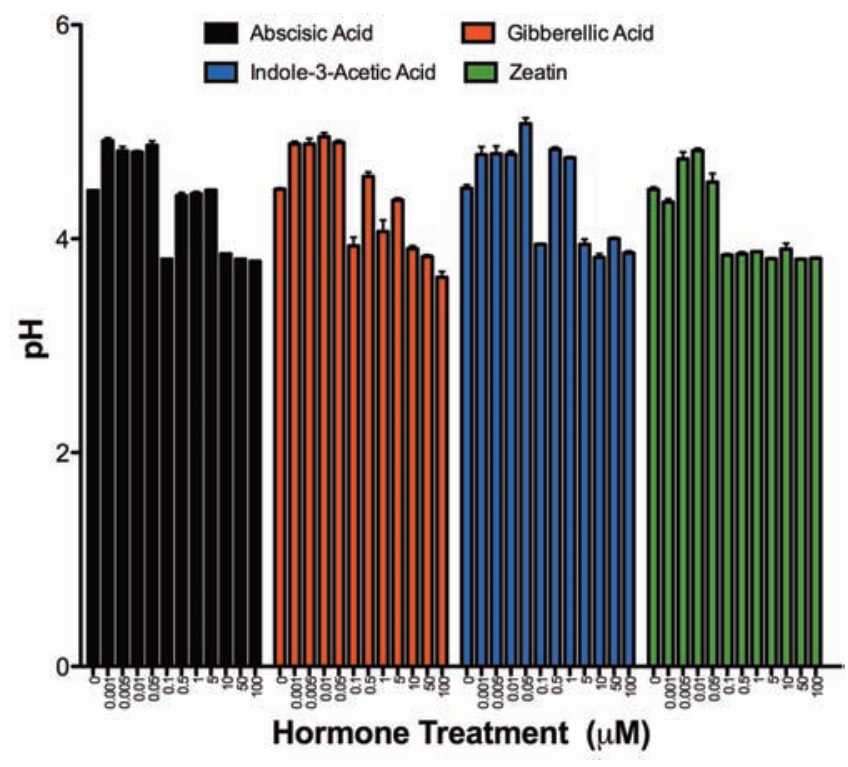

Figure 3. Phytohormones influence the final culture $\mathrm{pH}$ of $G$. xylinus. The supernatant $\mathrm{pH}$ of $\mathrm{G}$. xylinus cultures grown statically in the presence and absence of phytohormones for seven days at $30^{\circ} \mathrm{C}$ in Schramm-Hestrin broth were measured after pellicle removal. Data show the mean for three biological replicates each with six technical replicates. Error bars shown are the standard error of the mean.

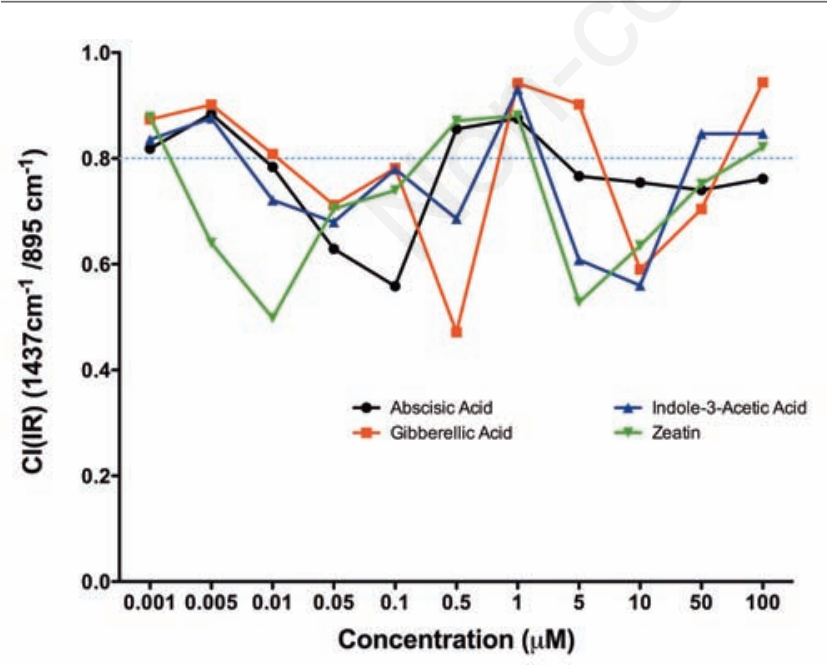

Figure 4. Growth in the presence of phytohormones influences $G$. xylinus pellicle crystallinity. The crystallinity index, CI (IR) for G. xylinus pellicles produced when grown for seven days under static conditions at $30^{\circ} \mathrm{C}$ in Schramm-Hestrin broth in the presence of the phytohoromones abscisic acid, gibberellic acid, indole-3-acetic acid, and zeatin. The blue dashed line shows the CI (IR) of untreated pellicles $(\mathbf{0 . 8 0})$ for comparison.

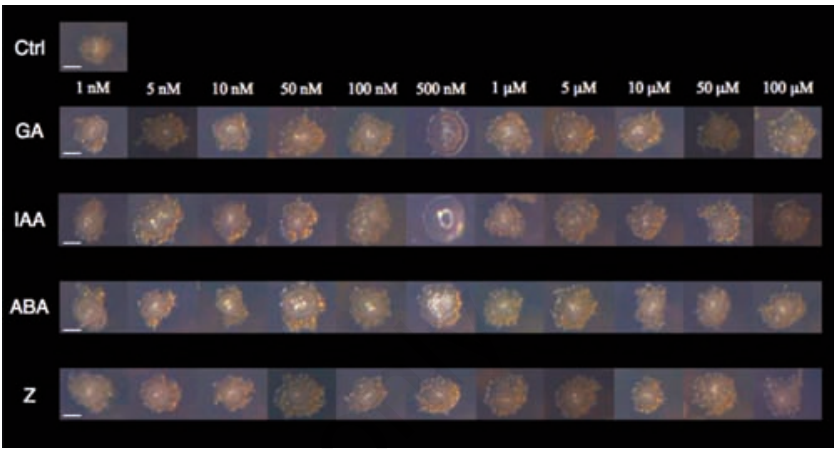

Figure 5. Phytohormones alter the colonial morphology of $G$. xylinus. Representative colonial morphologies exhibited by $G$. xylinus grown on Schramm-Hestrin agar at $30^{\circ} \mathrm{C}$ in the presence and absence of increasing concentrations of gibberellic acid (GA), idole-3-acetic acid (IAA), abscisic acid (ABA) and zeatin (Z). Photographs were taken after five days of growth. Scale bar represents $0.5 \mathrm{~cm}$.

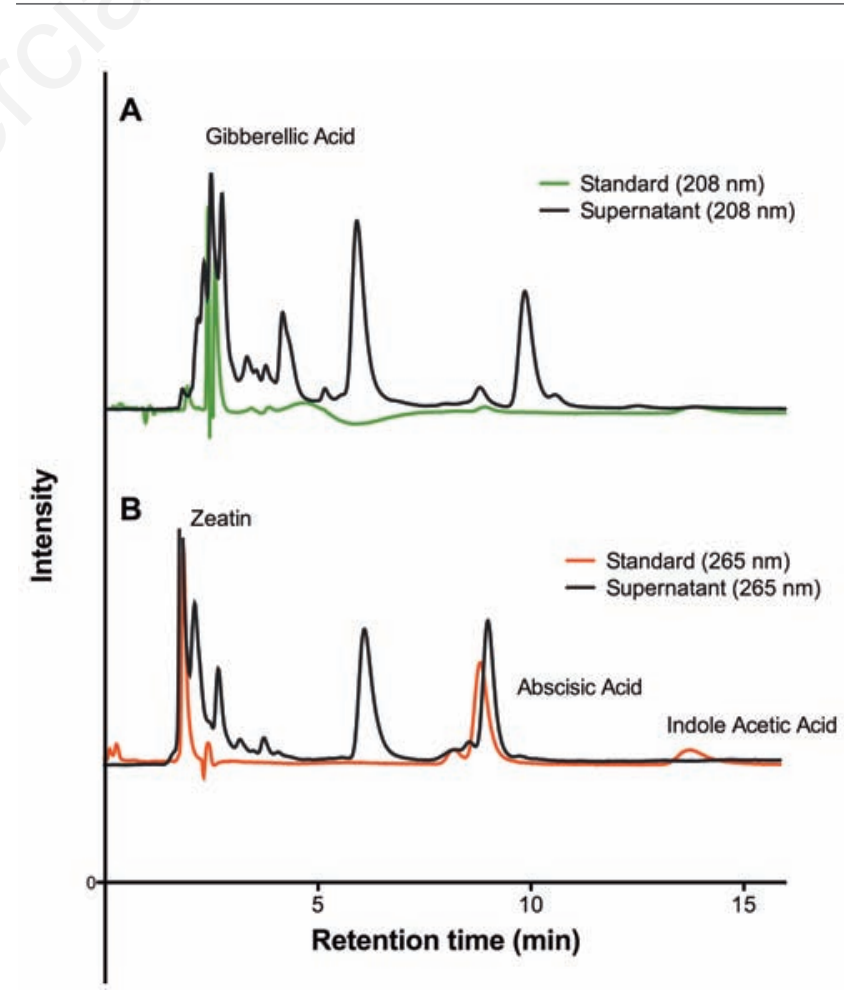

Figure 6. Gluconacetobacter xylinus produces endogenous phytohormones. High performance liquid chromatography of culture supernatant extracts showing endogenous $(\mathrm{A})$ gibberellic acid and endogenous (B) abscisic acid and zeatin production analyzed at the optimum wavelength for each hormone as described in the Materials and Methods section. No indole-3-acetic acid was detected in $G$. xylinus cultures. 
stimulation was hormone, bacterial strain and growth medium dependent. ${ }^{55}$ Interestingly, in the same study, the cytokinin, kinetin was found to inhibit Azospirillum growth at high concentrations. We did not observe any growth inhibition of $G$. xylinus by the cytokinin, $\mathrm{Z}$ used in this study.

The $\mathrm{pH}$ of culture supernatants was affected by phytohormone concentration. Lower $\mathrm{pHs}$ were observed for higher hormone concentrations (10 $\mu \mathrm{M}$ to $100 \mu \mathrm{M})$ compared to untreated cultures (Figure 3). Whether the $\mathrm{pH}$ differences observed were due to acetic acid production or due to excretion of other organic acids was not determined in this study.

Exogenous ABA increased the growth rate of $G$. xylinus (Figure 1). In general, pellicle wet weight (Figure 2B) was inversely proportional to the bacterial cellulose yield (Figure 2C) suggesting ABA influenced pellicle density and hydration. Pellicle crystallinity was moderately influenced by exogenous $\mathrm{ABA}$ with the lowest crystallinity index observed at a concentration of $100 \mathrm{nM}$ compared to untreated pellicles which exhibited a crystallinity index of 0.80 . The endogenous synthesis of ABA by G. xylinus (Figure 6) may serve to encourage fruit ripening. In grapes, the most important hormone involved in ripening is ABA. ${ }^{45} \mathrm{ABA}$ has been demonstrated to suppress resistance to necrotrophic pathogens. ${ }^{56}$ We speculate that the increase in cellulose yield observed for G. xylinus in response to this hormone shields its food source against other competitive microbes as has been demonstrated in apple-slice colonization studies. ${ }^{1}$

\section{Conclusions}

In conclusion, this research provides insight into the effects of phytohormones on the growth, cellulose production and cellulose structure in the model cellulose-producing bacterium $G$. xylinus. Furthermore, we report for the first time, endogenous production of GA, ABA and $\mathrm{Z}$ by G. xylinus. The results presented here have tremendous implications for understanding the cellulose synthesis of $G$. xylinus and identifying key factors that affect the interaction of this organism with plants.

\section{References}

1. Williams WS, Cannon RE. Alternative environmental roles for cellulose produced by Acetobacter xylinum. Appl Environ Microbiol 1989;55:2448-52.

2. Raspor P, Goranovi D. Biotechnological applications of acetic acid bacteria. Crit Rev Biotechnol 2008;28:101-24.

3. Chawla P, Bajaj I, Survase S, Singhal R. Microbial cellulose: Fermentative production and applications. Food Technol Biotechnol 2009;47:107-24.

4. Ross P, Aloni Y, Weinhouse C, et al. An unusual guanyl oligonucleotide regulates cellulose synthesis in Acetobacter xylinum. FEBS Lett 1985;186:191-6.

5. Czaja W, Krystynowicz A, Bielecki S, Malcolm Brown R. Microbial cellulose-the natural power to heal wounds. Biomaterials 2006;27:145-51.

6. El-Saied H, Basta AH, Gobran RH. Research progress in friendly environmental technology for the production of cellulose products (bacterial cellulose and its application). Polymer-Plastics Technol Eng 2004;43:797-820.

7. Kawano Y, Saotome T, Ochiai Y, et al. Cellulose accumulation and a cellulose synthase gene are responsible for cell aggregation in the cyanobacterium Thermosynechococcus vulcanus RKN. Plant Cell Physiol 2011;52:957-66.
8. Schramm M, Hestrin S. Factors affecting production of cellulose at the air/liquid interface of a culture of Acetobacter xylinum. J Gen Microbiol 1954;11:123-9.

9. Saxena IM, Brown RM. Identification of a second cellulose synthase gene (acsAII) in Acetobacter xylinum. J Bacteriol 1995; 177:5276-83.

10. Saxena IM, Kudlicka K, Okuda K, Brown RM. Characterization of genes in the cellulose-synthesizing operon (acs operon) of Acetobacter xylinum: implications for cellulose crystallization. J Bacteriol 1994;176:5735-52.

11. Yasutake Y, Kawano S, Tajima K, Yao M, et al. Structural characterization of the Acetobacter xylinum endo-beta-1,4-glucanase CMCax required for cellulose biosynthesis. Proteins 2006;64:1069-77.

12. Maneerung T, Tokura S, Rujiravanit R. Impregnation of silver nanoparticles into bacterial cellulose for antimicrobial wound dressing. Carbohydrate Polymers 2008;72:43-51.

13. Brown EE, Laborie M-PG, Zhang J. Glutaraldehyde treatment of bacterial cellulose/fibrin composites: impact on morphology, tensile and viscoelastic properties. Cellulose 2011;19:127-37.

14. Evans BR, O'Neill HM, Malyvanh VP, et al. Palladium-bacterial cellulose membranes for fuel cells. Biosensors Bioelectronics 2003;18:917-23.

15. Shah J, Brown RM. Towards electronic paper displays made from microbial cellulose. Appl Microbiol Biotechnol 2005;66:352-5.

16. Strap JL, Latos AJ, Shim I, Bonetta DT. Characterization of pellicle inhibition in Gluconacetobacter xylinum 53582 by a small molecule, pellicin, identified by a chemical genetics screen. PLoS ONE 2011;6:e28015.

17. Matthysse AG, Thomas DL, White AR. Mechanism of cellulose synthesis in Agrobacterium tumefaciens. J Bacteriol 1995;177:1076-81.

18. Matthysse AG, Marry M, Krall L, et al. The effect of cellulose overproduction on binding and biofilm formation on roots by Agrobacterium tumefaciens. Mol Plant Microbe Interact 2005;18:1002-10.

19. Rodríguez-Navarro DN, Dardanelli MS, Ruíz-Saínz JE. Attachment of bacteria to the roots of higher plants. FEMS Microbiol Lett 2007;272:127-36.

20. Barak JD, Jahn CE, Gibson DL, Charkowski AO. The role of cellulose and 0 -antigen capsule in the colonization of plants by Salmonella enterica. Mol Plant Microbe Interact 2007;20:1083-91.

21. Cosgrove DJ. Wall structure and wall loosening. A look backwards and forwards. Plant Physiol 2001;125:131-4.

22. Tanimoto E. Regulation of root growth by plant hormones-roles for auxin and gibberellin. Crit Rev Plant Sci 2005;24:249-65.

23. Tsavkelova EA, Klimova SY, Cherdyntseva TA, Netrusov AI. Hormones and hormone-like substances of microorganisms: A review. Appl Biochem Microbiol 2006;42:229-35.

24. Davies P. The plant hormones: their nature, occurrence, and functions. In: Davies P, ed. Plant hormones. Springer: Dordrecht; 2010. pp 1-15.

25. Sánchez-Rodríguez C, Rubio-Somoza I, Sibout R, Persson S. Phytohormones and the cell wall in Arabidopsis during seedling growth. Trends Plant Sci 2010;15:291-301.

26. Haberer G, Kieber JJ. Cytokinins. New Insights into a classic phytohormone. Plant Physiol 2002;128:354-62.

27. Skoog F, Armstrong DJ. Cytokinins. Annu Rev Plant Physiol 1970;21:359-84.

28. Davies C, Boss PK, Robinson SP. Treatment of grape berries, a nonclimacteric fruit with a synthetic auxin, retards ripening and alters the expression of developmentally regulated genes. Plant Physiol 1997;115:1155-61.

29. Zhang YL, Zhang RG. Effects of ABA content on the development of abscission zone and berry falling after harvesting of grapes. Agric Sci China 2009;8:59-67. 
30. Costacurta A, Vanderleyden J. Synthesis of phytohormones by plant-associated bacteria. Crit Rev Microbiol 1995;21:1-18.

31. Karadeniz A, Topcuo lu F, nan S. Auxin, Gibberellin, cytokinin and abscisic acid production in some bacteria. World $\mathrm{J}$ Microbiol Biotechnol 2006;22:1061-4.

32. Solans M, Vobis G, Cassán F, et al. Production of phytohormones by root-associated saprophytic actinomycetes isolated from the actinorhizal plant Ochetophila trinervis. World J Microbiol Biotechnol 2011;27:2195-202.

33. Tsavkelova EA, Klimova SY, Cherdyntseva TA, Netrusov AI. Microbial producers of plant growth stimulators and their practical use: a review. Appl Biochem Microbiol 2006;42:117-26.

34. Bastián F, Cohen A, Piccoli P, et al. Production of indole-3-acetic acid and gibberellins A 1 and A 3 by Acetobacter diazotrophicus and Herbaspirillum seropedicae in chemically-defined culture media. Plant Growth Regulation 1998;24:7-11.

35. Lee S, Kennedy C. Characterization of indole-3-acetic acid (IAA) produced by the sugarcane endophyte Acetobacter diazotrophicus, in sugarcane growth. In: Pedrosa F0, Hungria M, Yates G, Newton WE, eds. Nitrogen fixation: From molecules to crop productivity. Springer: Dordrecth; 2002. pp 421-1.

36. Bae S, Sugano Y, Shoda M. Improvement of bacterial cellulose production by addition of agar in a jar fermentor. $\mathrm{J}$ Biosci Bioeng 2004;97:33-8.

37. Czaja W, Romanovicz D, Brown R Jr. Structural investigations of microbial cellulose produced in stationary and agitated culture. Cellulose 2004;11:403-11.

38. Ünyar S, Topcuo lu F, Ünyayar A. A modified method for extraction and identification of indole-3-acetic acid (IAA), gibberellic acid (GA3), abscisic acid (ABA) and zeatin produced by Phanerochaete chyrsosporium ME 446. Bulgarian J Plant Physiol 1996;22:105-10.

39. Kelen M, Demiralay EC, Sen S, Ozkan G. Separation of abscisic acid, indole-3-acetic acid, gibberellic acid in $99 \mathrm{R}$ (Vitis berlandieri $x$ Vitis rupestris) and rose oil (Rosa damascena Mill.) by reversed phase liquid chromatography. Turkish J Chem 2004;28:603-10.

40. Gordon SA, Weber RP. Colorimetric estimation of indoleacetic acid. Plant Physiol 1951;26:192-5.

41. Sarwar M, Kremer RJ. Determination of bacterially derived auxins using a microplate method. Lett Appl Microbiol 1995;20:282-5.

42. de Salamone IEG, Hynes RK, Nelson LM. Cytokinin production by plant growth promoting rhizobacteria and selected mutants. Can J Microbiol 2001;47:404-11.

43. Tien TM, Gaskins MH, Hubbell DH. Plant growth substances produced by Azospirillum brasilense and their effect on the growth of pearl millet (Pennisetum americanum L.). Appl Environ Microbiol 1979;37:1016-24.

44. Gan S. The hormonal regulation of senescence. In: Davies P, ed. Plant hormones. Springer: Dordrecht; 2010. pp 597-617.

45. Coombe BG, Hale CR. The hormone content of ripening grape berries and the effects of growth substance treatments. Plant Physiol 1973;51:629-34.

46. Bastías A, López-Climent M, Valcárcel M, et al. Modulation of organic acids and sugar content in tomato fruits by an abscisic acid-regulated transcription factor. Physiol Plant 2011;141:215-26.

47. Fuentes-Ramirez L, Jimenez-Salgado T. Acetobacter diazotrophicus, an indoleacetic acid producing bacterium isolated from sugarcane cultivars of Mexico. Plant Soil 1993;154:145-50.

48. Cohen JD. In vitro tomato fruit cultures demonstrate a role for indole-3-acetic acid in regulating fruit ripening. J Am Soc Horti Sci1996;121:520-4.

49. Tingwa P0, Young RE. The effect of indole-3-acetic acid and other growth regulators on the ripening of avocado fruits. Plant Physiol 1975;55:937-40.

50. Vendrell M. Reversion of senescence: effects of 2, 4-dichlorophenoxyacetic acid and indoleacetic acid on respiration, ethylene production, and ripening of banana fruit slices. Aust J Biol Sci 1969;22:601-10.

51. Jia H-F, Chai Y-M, Li C-L, et al. Abscisic acid plays an important role in the regulation of strawberry fruit ripening. Plant Physiol 2011;157:188-99.

52. Leveau JHJ, Lindow SE. Utilization of the plant hormone indole-3acetic acid for growth by Pseudomonas putida strain 1290. Appl Environ Microbiol 2005;71:2365-71.

53. Gravel V, Antoun H, Tweddell RJ. Growth stimulation and fruit yield improvement of greenhouse tomato plants by inoculation with Pseudomonas putida or Trichoderma atroviride: Possible role of indole acetic acid (IAA). Soil Biol Biochem 2007;39:1968-77.

54. Taiz L, Honigman WA. Production of cell wall hydrolyzing enzymes by barley aleurone layers in response to gibberellic acid. Plant Physiol 1976;58:380.

55. Strzelczyk E, Kampert M, Rozycki H, Li CY. Effect of plant growth hormones on growth of Azospirillum sp. in media with different carbon sources. Acta Microbiol Pol 1994;43:89-9.

56. Fan J, Hill L, Crooks C, et al. Abscisic acid has a key role in modulating diverse plant-pathogen interactions. Plant Physiol 2009;150:1750-61. 